\title{
Detecting ALS and Parkinson's disease in rats through locomotion analysis
}

\author{
Wenlong Tang $\cdot$ Paul Yarowsky $\cdot$ Uri Tasch
}

Received: 17 January 2012/Revised: 6 February 2012/ Accepted: 8 February 2012/Published online: 14 March 2012

(c) Springer-Verlag 2012

\begin{abstract}
We describe a method that detects locomotion deficiencies due to amyotrophic lateral sclerosis (ALS) and Parkinson's disease (PD) in laboratory rats. The locomotion deficiencies are recognized by performing three different comparisons: (1) comparing the locomotion of an ALS model, G93A mutation of SOD1 with control rats, (2) comparing the locomotion of a PD model, 6-OHDA lesioned with control rats, and (3) comparing the locomotion of G93A/SOD1 and 6-OHDA lesioned rats. Each comparison resulted in different set of locomotion parameters for ALS and PD that characterized the locomotion deficiencies and resulted in the best logistic regression model that classifies the rats into diseased/healthy groups with minimum error. The sensitivity and the specificity of the classification for comparisons (1), (2), and (3) were above $90 \%$.
\end{abstract}

Keywords ALS · PD - Rats - Locomotion analysis · Logistic regression model

\section{Introduction}

Increasing number of individuals are being affected by neurodegenerative diseases, such as amyotrophic lateral

\footnotetext{
W. Tang $\cdot$ U. Tasch $(\bowtie)$

Department of Mechanical Engineering,

University of Maryland Baltimore County,

1000 Hilltop Circle, Baltimore, MD 21250, USA

e-mail: tasch@umbc.edu

P. Yarowsky

Department of Pharmacology and Experimental Therapeutics, University of Maryland School of Medicine and the Veterans Administration, Baltimore, MD 21201, USA
}

sclerosis (ALS) and Parkinson's disease (PD), which creates a growing need for effective diagnosis and treatment protocols (Liao et al. 2008). It is reported that as many as 30,000 individuals in the US suffer from ALS and 5,000 more individuals are estimated to be diagnosed every year. Cases of PD give an overall annualized age- and genderadjusted incidence rate of 13.4 per 100,000 (Van Den Eeden et al. 2003). It is the second most common neurodegenerative disorder (Kasten et al. 2007).

Animal models are instrumental for investigating neurological diseases involving motor function in humans. We have studied two such models in rats: an ALS model with mutant superoxide dismutase 1 (SOD1), the G93A mutation, and a rat model of $\mathrm{PD}$, the unilateral 6-hydroxydopamine (6-OHDA) lesioned animal (Jackson et al. 2002; Ries and Burke 2008).

This manuscript presents a methodology that uses a locomotion analysis system measuring ground reaction forces in three dimensions (Tasch et al. 2008; Tasch 2010) to detect whether the gait of the animal is affected by ALS (Tang et al. 2009) and/or PD (Tang et al. 2010) at an early pre-symptomatic stage. The G93A/SOD1 is a genetic model of the disease, yet the current detection techniques (Dunlop et al. 2003) detect the presence of the disease only between 8 and 10 weeks of age; the 6-OH PD model is environmental toxin model of disease and behavioral symptoms are seen 3 weeks after the lesion (McCoy et al. 2008).

Amende et al. (2005) found the mouse models of PD, MPTP (1-methyl-4-phenyl-1, 2, 3, 6-tetrahydropyridine), have demonstrated significant gait disturbances, such as, shortened stride length, increased stride frequency, and increased stride-to-stride variability. However, the work does not provide any model to govern the gait disturbances they found. In this paper, we present three statistical 
models to detect changes in the locomotion that are attributed to the presence of either ALS or PD in rats, and to test the models' capabilities to discriminate control rats and the rats that are affected by ALS, control and 6-OHDA lesioned rats, and finally discriminate ALS and 6-OHDA lesioned rats.

\section{Methods and materials}

\subsection{Statistical modeling and validation}

Tasch et al. $(2008,2010)$ introduced a locomotion analysis system that measures ground reaction forces (GRFs) in vertical, longitudinal and transverse directions, as well as spatial (longitudinal and transverse) limb positions. Twenty locomotion parameters (LPs) per limb were derived from the GRFs and spatial data which are synchronized with the images of the animal as it walks through the system.

These LPs are used as input to a logistic regression model that evaluates the predictions that the locomotion of the tested animal is affected by either ALS or PD. The mathematical expression of the logistic regression prediction is

$P($ a rat $\in$ diseased group $)=\frac{\exp \left(\beta_{0}+\sum \beta_{i} \times L P_{i}\right)}{1+\exp \left(\beta_{0}+\sum \beta_{i} \times L P_{i}\right)}$,

where $\beta_{0}$ is the intercept and $\beta_{i}$ is the $i$-th coefficient of the logistic regression model and is estimated by appropriate statistical methods. It was demonstrated that the accuracy of the predictions of these models is significantly improved when the LPs are transformed via spline transformations (Liu et al. 2009). An implementation of these transformations is available in SAS (PROC TRANSREG) (SAS Institute 2004). The logistic regression predictions are therefore expressed as:

$P($ a rat $\in$ diseased group $)=\frac{\exp \left(\beta_{0}+\sum \beta_{i} \times T L P_{i}\right)}{1+\exp \left(\beta_{0}+\sum \beta_{i} \times T L P_{i}\right)}$.

where TLPs are transformed LPs. This logistic regression model was used to derive the prediction models of ALS and PD in SOD1-G93A and 6-OHDA lesioned rats, respectively. The models were developed in three different ways: (1) comparing the locomotion of SOD1-G93A rats to the locomotion of controls, (2) comparing the locomotion of 6-OHDA lesioned and control rats, and (3) comparing the locomotion of SOD1-G93A and 6-OHDA lesioned rats. These three comparisons result in three distinct models that best capture the gait deficiencies when performing these comparisons.
A cross-validation method, known as leave-one-out (Efron and Tibshirani 1993), was employed to evaluate the performances of the three models and the sensitivity and specificity values of these three models were found to be better than $90 \%$.

\subsection{Obtaining locomotion measurements}

In comparison (1), four SOD1-G93A male rats and four Sprague-Dawley (SD) control male rats were used to model the locomotion deficiencies between ALS and control healthy rats. After 1 week of training for adaptation to the system, the eight rats walked through the locomotion analysis system (Tang et al. 2009) twice a week up to three times per day for a total of 8 weeks. A total of 163 test runs were recorded (96 runs from the SOD1-G93A rats and 67 runs from control SD rats).

To model locomotion deficiencies due to PD, four Sprague-Dawley (SD) control rats traversed through the locomotion analysis system (Tang et al. 2010) after 1 week of training for 10 days, twice per week and up to three times per day. After the tenth day, 6-OHDA was injected to the right medial forebrain bundle (MFB), following the procedure documented by Muir and Whishaw (1999). The rats recovered in 3 days and traversed through the locomotion system for additional 20 days after being exposed to the 6-OHDA toxin. By comparing the locomotion of the rats before and after being exposed to the 6-OHDA, one obtains the model that captures the locomotion deficiencies between Parkinsonian and control rats, which is comparison (2). Finally, the locomotion of the SOD1 and Parkinsonian rats were compared and a third model that captures the locomotion differences between SOD1 and 6-OHDA lesioned rats (comparison (3)) was established.

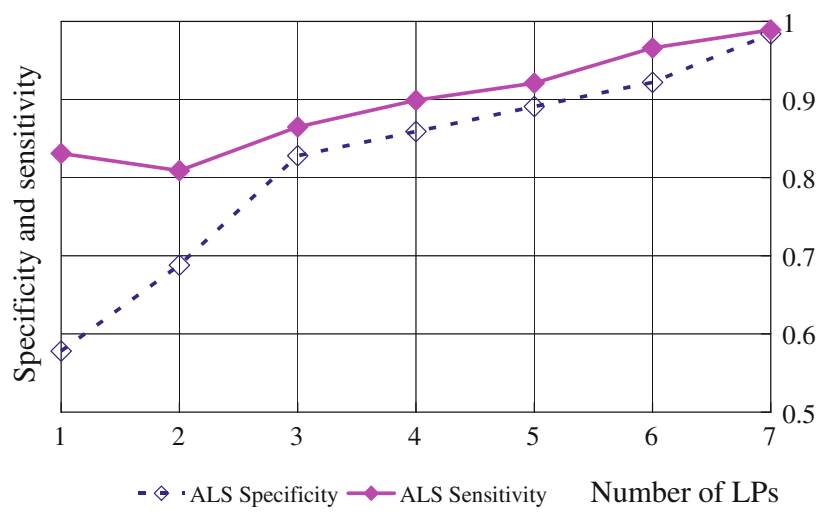

Fig. 1 Sensitivity and specificity versus the number of best performing locomotion parameters (LPs) used to model the locomotion deficiencies of the SOD1-G93A rats. Best performing LPs result in the lowest misclassification errors when the cutoff value is set to 0.5 . Note the improved specificity and sensitivity values are more than 0.98 as the number of best performing LPs increases to 7 


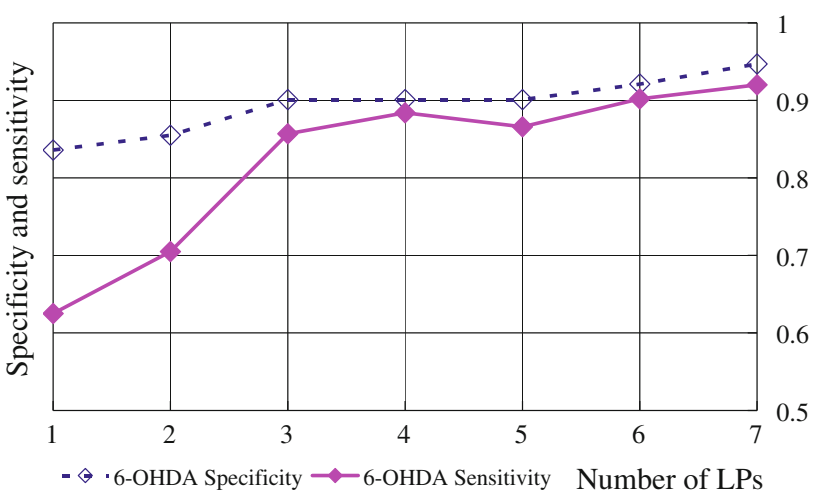

Fig. 2 Sensitivity and specificity versus the number of best performing locomotion parameters (LPs) used to model the locomotion deficiencies of the 6-OHDA lesioned rats. Best performing LPs result in the lowest misclassification errors when the cutoff value is set to 0.5 . Note the improved specificity and sensitivity values are more than 0.92 as the number of best performing LPs increases to 7

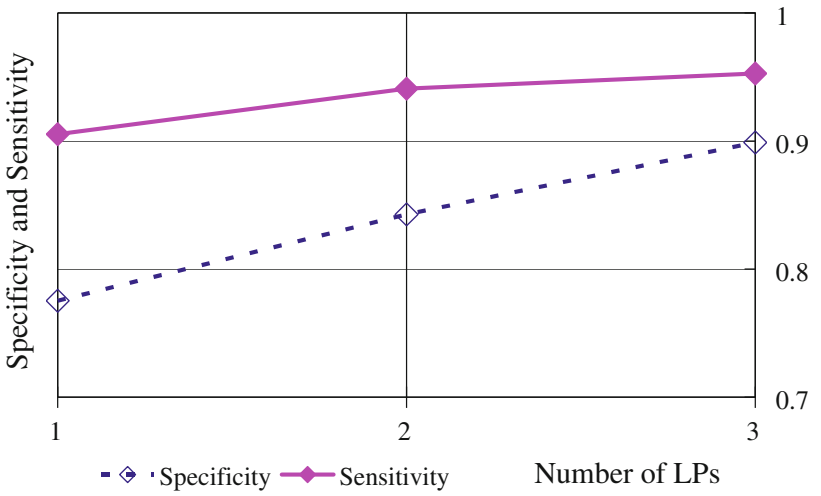

Fig. 3 Sensitivity and specificity versus the number of best performing locomotion parameters (LPs) used to distinguish the locomotion of the SOD1-G93A and the 6-OHDA lesioned rats. Best performing LPs result in the lowest misclassification errors when the cutoff value is set to 0.5 . Note the improved specificity and sensitivity values are above 0.90 as the number of best performing LPs increases to 3

\subsection{Animal protocols}

All protocols described in this study were approved by the IACUC at the University of Maryland, Baltimore County and the University of Maryland, School of Medicine. At the end of the experiments, animals were euthanized by sodium pentobarbitol injection (150 mg/kg IP).

\section{Results}

To derive the three logistic regression models that best capture the locomotion differences between (1) SOD1 and control rats, (2) 6-OHDA lesioned and control rats, and (3) SOD1 and 6-OHDA lesioned rats, the sensitivity (the percentage of treated rats which are correctly identified as having the condition), and specificity (the percentage of control rats which are correctly identified as not having the condition) of the three models were evaluated when the number of LPs that were used to derive the logistic regression models was evaluated. Figures 1, 2, and 3 depict the sensitivity and specificity values of models (1), (2) and (3) as the number of LPs used to derive the logistic regression models are increased.

The LPs were ranked according to their individual abilities to distinguish between any two different groups. Locomotion parameters with smaller misclassification error obtained higher ranking. The seven LPs that best distinguish between the locomotion of SOD1 and control rats are listed in Table 1, with sensitivity and specificity values of 0.99 and 0.98 , respectively (Fig. 1). The locomotion of the 6-OHDA lesioned and control rats is best distinguished when the seven LPs listed in Table 2 are used with sensitivity and specificity values of 0.92 and 0.95 , respectively (Fig. 2). Note that all the LPs that best capture the locomotion differences between the case and control rats in the above two experiments are derived from GRFs measurements. This demonstrates the importance of measuring GRFs components to detect locomotion deficiencies of SOD1 rats. Finally, the three LPs that best distinguish between the locomotion of the SOD1 and the 6-OHDA rats are listed in Table 3, with sensitivity and specificity values of 0.95 and 0.90 , respectively (Fig. 3). Note that two ( $F y_{\omega}$ and $\left.F y_{\min }\right)$ out of the three LPs that best capture the

Table 1 The LPs that best distinguish the SOD1 and the control rats

\begin{tabular}{|c|c|c|c|}
\hline & LPs & $\begin{array}{l}\text { Misclassification } \\
\text { rate of LP (\%) }\end{array}$ & Definition \\
\hline \multirow[t]{7}{*}{ ALS model } & $F y B$ & 18.4 & Mean value of the braking(negative) longitudinal force of a selected limb \\
\hline & $T \_F y_{\max }$ & 20.3 & Time of $F y_{\max }$ divided by stance time \\
\hline & $F y_{\max }$ & 24.5 & Maximum value of the longitudinal GRF component of a selected paw \\
\hline & $F z_{\omega}$ & 25.2 & The Fourier transform of $\mathrm{Fz}$ summed over the first $50 \mathrm{~Hz}$ for a selected $\operatorname{limb}\left[\int_{50} \mathrm{Fz} d \omega\right]$ \\
\hline & $F z_{\text {mean }}$ & 25.8 & The mean value of the vertical GRF component of a selected limb $\left[\frac{\int_{\text {s.Time }} F z \mathrm{~d} t}{\text { Stancetime }}\right]$ \\
\hline & $F y_{\omega}$ & 25.8 & The Fourier transform of Fy summed over the first $50 \mathrm{~Hz}$ for a selected paw $\left[\int_{50}\right.$ Fy $\left.d \omega\right]$ \\
\hline & NP & 25.8 & The number of samples in which the longitudinal force is propelling; the sampling rate is $200 \mathrm{~Hz}$ \\
\hline
\end{tabular}


Table 2 The $L P$ s that best distinguish the 6-OHDA lesioned and the control rats

\begin{tabular}{|c|c|c|c|}
\hline & LPs & $\begin{array}{l}\text { Misclassification } \\
\text { rate of LP }(\%)\end{array}$ & Definition \\
\hline \multirow{7}{*}{$\begin{array}{l}\text { Parkinson's disease } \\
\text { model }\end{array}$} & $F y_{\min }$ & 23.5 & Minimum value of the longitudinal GRF component of a selected paw \\
\hline & NP & 24.6 & $\begin{array}{l}\text { The number of samples in which the longitudinal force is propelling; the sampling rate is } \\
200 \mathrm{~Hz}\end{array}$ \\
\hline & $F x_{\max }$ & 24.6 & Maximum value of the transverse GRF component of a selected paw \\
\hline & $F y_{\text {mean }}$ & 25.4 & The average value of the longitudinal GRF component of a selected paw $\left[\frac{\int_{\text {S.Time }} F y \mathrm{~d} t}{\text { Stancetime }}\right]$ \\
\hline & Sym_FyP & 28.4 & $\begin{array}{l}\text { The symmetry of FyP, FyP is the mean value of the propelling (positive) longitudinal } \\
\text { force of a selected limb }\end{array}$ \\
\hline & $F y_{\max }$ & 29.2 & Maximum value of the longitudinal GRF component of a selected paw \\
\hline & NB & 30.3 & $\begin{array}{l}\text { The number of samples in which the longitudinal force is braking (negative in value); the } \\
\text { sampling rate is } 200 \mathrm{~Hz}\end{array}$ \\
\hline
\end{tabular}

Table 3 The LPs that best distinguish the SOD1 and the 6-OHDA lesioned rats

\begin{tabular}{llll}
\hline & LPs & $\begin{array}{l}\text { Misclassification } \\
\text { rate of LP }(\%)\end{array}$ & Definition \\
\hline $\begin{array}{l}\text { Model to distinguish ALS and } \\
\text { Parkinson's disease }\end{array}$ & $F y \omega$ & 13.6 & $\begin{array}{l}\text { The Fourier transform of Fy summed over the first } 50 \mathrm{~Hz} \text { for a selected } \\
\text { paw }\left[\int_{50} F y d \omega\right]_{A L S} \\
\text { Stride length of a selected limb calculated as the difference between two } \\
\text { consecutive contact positions; the contact positions are normalized by the floor } \\
\text { length, which is } 13.25 \text { inch } \\
\text { Minimum value of the longitudinal GRF component of a selected paw PD }\end{array}$ \\
\hline$F y_{\text {min }}$ & 15.5 & 14.7 &
\end{tabular}

Fig. 4 Probability of ALS for the four SOD1 (S1-S4) and 4 control $(C 1-C 4)$ rats versus days of the animals from their births. Each data point is an average of at least two predictions, calculated by the 7-LP model. Note that $P$ (ALS) for SOD1 and control rats is close to 1.0 and 0.0 , respectively, in all days except for a single day for rat $\mathrm{C} 4$

\section{S1 \& C1}

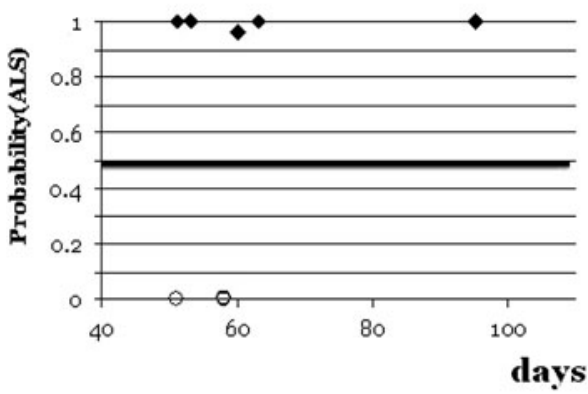

S3 \& C3

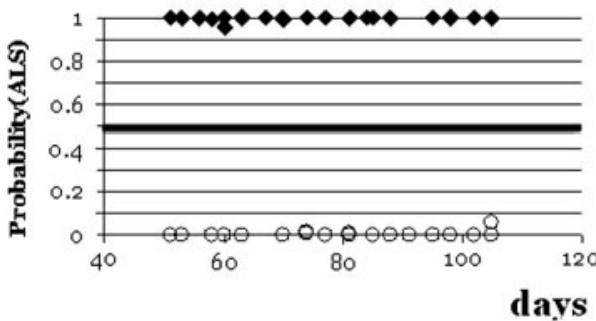

S2 \& C2

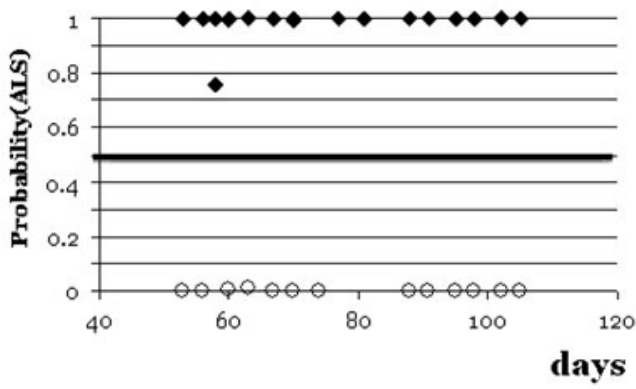

S4 \& C4

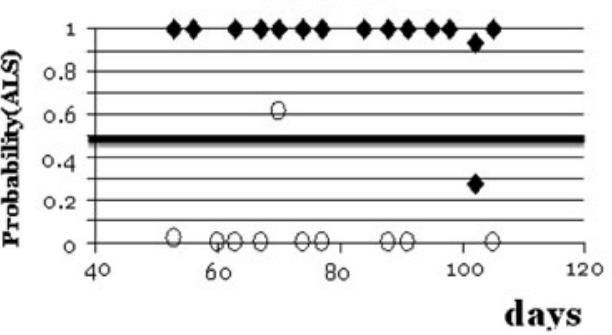

- SOD1-Gra3A O Control

locomotion differences between SOD1 and 6-OHDA rats are derived from GRFs measurements. This demonstrates again the importance of the GRFs measurements when characterizing locomotion. Further, LPs derived from vertical GRFs direction are not part of the LPs listed in either Table 2 or Table 3, and hence do not contribute to the characterization of the locomotion differences between 6-OHDA lesioned rats and control, and 6-OHDA and 
SOD1 rats. This is in agreement with the finding that in advanced Parkinsonian rats traverse while dragging their limbs (Metz et al. 2005).

Models (1), (2) and (3) were validated by the leave-oneout method. Figure 4 depicts the probabilities that SOD1 and control rats possess locomotion deficiencies due to ALS. Note that the high probability values $(>0.5)$ for the SOD1 rats and low probability values $(<0.5)$ for the control rats.

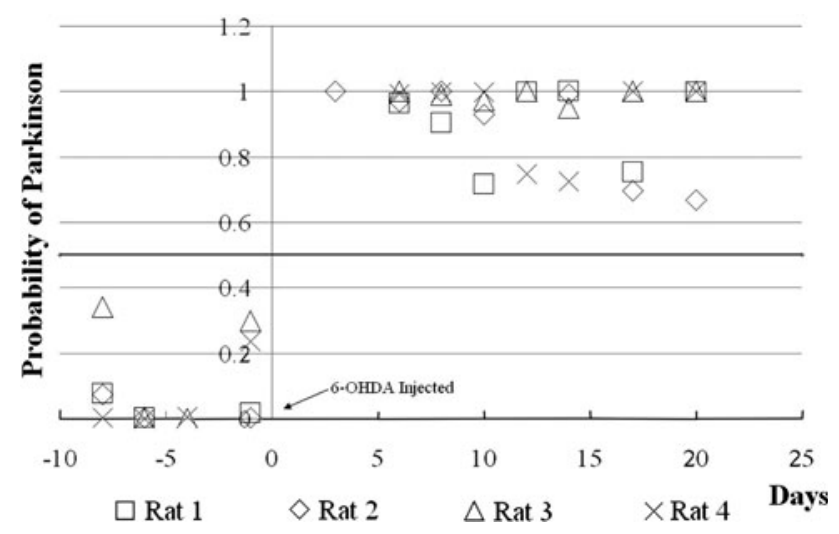

Fig. 5 Probability of Parkinson for the four rats versus days for the animals that traversed through the locomotion analysis system. The 6-OHDA injection occurs on the day 0 . Each data point is an average of at least two predictions, calculated by the 7-LP model. Note that all the data points are below 0.5 before the injection and above 0.5 after the injection (day 0)
Similarly, Fig. 5 depicts the probabilities that a rat is Parkinsonian and it is apparent that the SD control rats possess low probabilities $(<0.5)$ before the 6-OHDA injection (day 0 ), and in contrary possess high probability $(>0.5)$ after the day 6-OHDA is injected (day 0$)$.

Finally, Fig. 6 depicts the classification of the four SOD1 and four 6-OHDA rats into ALS and PD groups based on each individual test run. The distance from the ALS and PD horizontal lines represents the probability that a rat does not belong to either an ALS or PD group, respectively. Based on a cutoff probability value of 0.5 , the four SOD1 and four 6-OHDA rats are classified correctly except for a single misclassification of S1 on the tenth day. Each data point of probability in the description above is an average of at least two predictions, calculated by the statistical models.

\section{Conclusions and discussions}

This manuscript introduces an effective methodology to derive logistic regression models that characterize locomotion deficiencies due to neurological diseases, such as PD and ALS. The locomotion deficiencies are captured by measuring parameters in four domains: force, distance, time and frequency. A number of LPs were generated from the collected data and several selected LPs were used to calculate the probability that the observed locomotion
Fig. 6 Four SOD1-G93A (S1-S4) and four 6-OHDA lesioned (PD1-PD4) versus time. For each test run the 8 rats are grouped as ALS and PD. The distance from the ALS and $\mathrm{PD}$ horizontal lines represent the probability that a rat, based on a particular test run does not belong to the ALS or PD group, i.e., when the mark is on the PD or the ALS line that rat belongs to the 6-OHDA or SOD1 group, respectively. Each data point is an average of at least two predictions, calculated by the 3-LP model. Note that based on a cutoff value of 0.5 , the eight rats are classified correctly, except to a single misclassification of $\mathrm{S} 1$ on the tenth day
S1 \& PD1

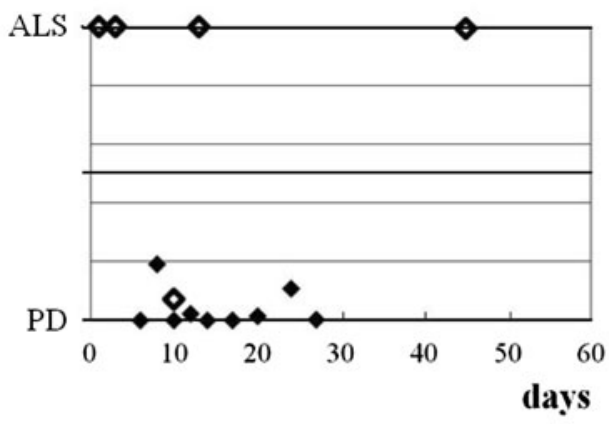

S3 \& PD3

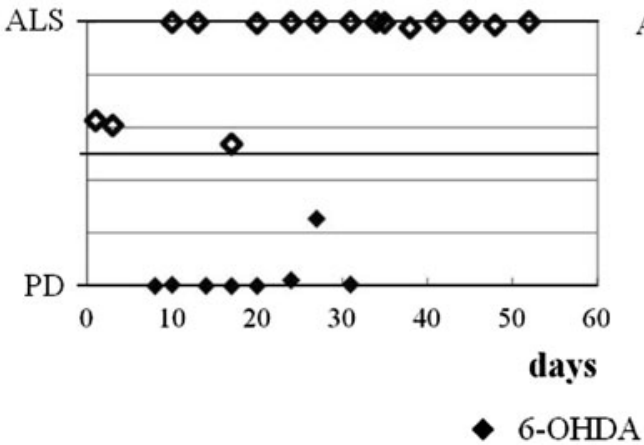

S2 \& PD2

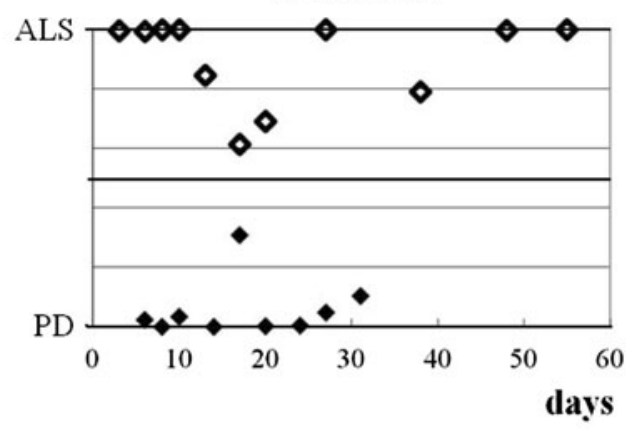

S4 \& PD4

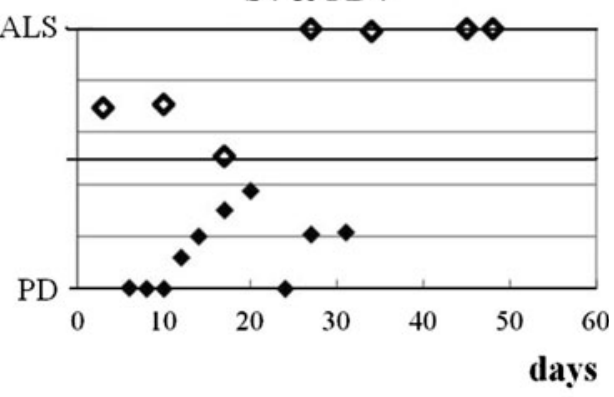


deficiencies are due to a certain disease, i.e. ALS or PD. Three comparisons were performed (1) SOD1 and control rats, (2) 6-OHDA lesioned rats and control, and (3) SOD1 and 6-OHDA lesioned rats.

Three different statistical models were developed and tested. The inputs of the statistical models are the values of selected LPs that best capture the change in locomotion due to a specific disease. The criterion of the LP selection is the performance of distinguishing between the two groups of rats, respectively. Model performance has two measures: sensitivity and specificity. All the three models reach sensitivity and specificity values of better than $90 \%$.

Comparing to other locomotion analysis (Hampton et al. 2011; Amende et al. 2005), the advantage of the proposed methodology is to build statistical models to detect disorders (e.g., PD, ALS), rather than finding disturbance in some locomotion parameters. Statistical models are able to provide quantitative detecting probability that other locomotion analysis cannot deliver.

Acknowledgments The authors deeply appreciate the anonymous referees for their valuable suggestions. The contributions of Nagaraj K. Neerchal, Kim McDowell and Mark Limsam are acknowledged. Support was provided by the Veterans Administration (PY).

\section{References}

Amende I, Kale A, McCue S, Glazier S, Morgan JP, Hampton TG (2005) Gait dynamics in mouse models of Parkinson's disease and Huntington's disease. J Neuro Eng Rehab 2:20. doi: 10.1186/1743-0003-2-20

Dunlop J, Beal McIlvain H, She Y, Howland DS (2003) Impaired spinal cord glutamate transport capacity and reduced sensitivity to riluzole in a transgenic superoxide dismutase mutant rat model of amyotrophic lateral sclerosis. J Neurosci 23:1688-1696

Efron B, Tibshirani RJ (1993) An introduction to the bootstrap. Chapman and Hall, New York

Hampton T, Kale A, Amende I, Tang W, McCue S, Bhagavan H, VanDongen C (2011) Gait disturbances in dystrophic hamsters. J Biomed Biotechnol. doi: 10.1155/2011/235354
Jackson M, Ganel R, Rothstein JD (2002) Models of amyotrophic lateral sclerosis. Curr Protoc Neurosci 9:13

Kasten M, Chade A, Tanner CM (2007) Epidemiology of Parkinson's disease. Handb Clin Neurol 83:129-151

Liao F, Wang J, He P (2008) Multi-resolution entropy analysis of gait symmetry in neurological degenerative diseases and amyotrophic lateral sclerosis. Med Eng Phys 30:299-310

Liu J, Neerchal NK, Tasch U, Dyer RM, Rajkondawar PG (2009) Enhancing the predictions accuracy of bovine lameness models through nonlinear transformations of the limb movement variables. J Dairy Sci 92:2539-2550

McCoy MK, Ruhn KA, Martinez TN, McAlpine FE, Blesch A, Tansey MG (2008) Intranigral lentiviral delivery of dominantnegative TNF attenuates neurodegeneration and behavioral deficits in hemiparkinsonian rats. Am Soc Gene Ther 16:1572-1579

Metz GA, Tse A, Ballermann M, Smith LK, Fouad K (2005) The unilateral 6-OHDA rat model of Parkinson's disease revisited: an electromyographic and behavioural analysis. Eur J Neurosci 22:735-744

Muir GD, Whishaw IQ (1999) Ground reaction forces in locomoting hemi-parkinsonian rats: a definitive test for impairments and compensations. Exp Brain Res 126:307-314

Ries V, Burke RE (2008) Rodent toxin models of PD: an overview. In: Nass R, Przedborski S (eds) Parkinson's disease: molecular and therapeutic insights from model systems. Elsevier pp 135-146

SAS Institute (2004) SAS/STAT 9.1 User's guide. SAS Institute, Cary

Tang W, Tasch U, Neerchal NK, Zhu L, Yarowsky P (2009) Measuring early pre-symptomatic changes in locomotion of SOD1-G93A rats: a rodent model of amyotrophic lateral sclerosis. J Neurosci Methods 176:254-262

Tang W, McDowell K, Limsam M, Neerchal NK, Yarousky P, Tasch U (2010) Locomotion analysis of Sprague-Dawley rats before and after injecting 6-OHDA. Behav Brain Res 210:131-133

Tasch U (2010) Diagnosis system and method. Pending US Patent No. US2010/0217157 A1

Tasch U, Moubarak P, Tang W, Zhu L, Lovering RM, Roche JA, Bloch RJ (2008) An instrument that simultaneously measures spatial gait parameters and ground reaction forces of locomoting rats. In: Proceedings of the ninth biennial ASME conference on engineering systems design and analysis ESDA08

Van Den Eeden SK, Tanner CM, Bernstein AL, Fross RD, Leimpeter A, Bloch DA et al (2003) Incidence of Parkinson's disease: variation by age, gender, and race/ethnicity. Am J Epidemiol 157:1015-1022 